\title{
Caracterização florística e chave dendrológica para espécies em área de Cerradão na transição Cerrado-Pantanal, Mato Grosso, Brasil
}

\author{
Otávio Miranda Verly ${ }^{1,2, *}$ (1), Simone Silva' (1), Reginaldo Antonio Medeiros ${ }^{2}$ (1), Antonio Miguel \\ Olivo-Neto ${ }^{3}$ (1) , Carlos Alberto Ramos Domiciano ${ }^{1,2}$ (D), Poliane Rodrigues Rosa ${ }^{2}$ (D)
}

\begin{abstract}
* verly.miranda@gmail.com
1 Universidade Federal de Viçosa, Departamento de Engenharia Florestal, Programa de Pós-Graduação em Ciência Florestal, Avenida Peter Henry Rolfs, s/n, Campus Universitário, 36570-900, Viçosa, Minas Gerais, Brasil.

${ }_{2}^{2}$ Instituto Federal de Educação, Ciência e Tecnologia de Mato Grosso - Campus Cáceres - Prof. Olegário Baldo, Avenida Europa, n³000, Vila Real, 78201-382, Cáceres, Mato Grosso, Brasil.

3Universidade do Estado de Mato Grosso, Programa de Pós-Graduação em Ciências Ambientais, Herbário do Pantanal "Vali Joana Pott" - HPAN, Avenida Santos Dumont, s/n, Cidade Universitária, Bloco II, DNER, 78200-000, Cáceres, Mato Grosso, Brasil.
\end{abstract}

Recebido em 31.1.2020

Aceito em 24.V.2021

DOI 10.21826/2446-82312021v76e2021010

RESUMO - O estudo objetivou a catalogação e a caracterização das espécies arbóreo-arbustivas em um fragmento de Cerradão, a fim de confeccionar uma chave dicotômica baseada em características dendrológicas para identificação das espécies da região. A área está localizada na transição CerradoPantanal, em Cáceres, MT, e possui 3 hectares que foram divididos em 32 parcelas de $48 \times 20 \mathrm{~m}$. O levantamento foi censitário, inventariando os indivíduos com diâmetro $\geq 5,0 \mathrm{~cm}$ a $30 \mathrm{~cm}$ do solo. A chave foi elaborada a partir da compilação dos caracteres dendrológicos observados e caracterizados. Foram amostradas 84 espécies, pertencentes a 73 gêneros e 34 famílias. As famílias Fabaceae ( 21 espécies), Malvaceae (6 espécies), Anacardiaceae/Bignoniaceae (5 spp. cada), Rubiaceae (4 spp.) e Euphorbiaceae/Vochysiaceae (3 spp. cada) contabilizaram 55,95\% da riqueza de espécies. Entre os gêneros, Bauhinia se destacou com três espécies. O fragmento possui alta riqueza específica, e mesmo com a maioria dos componentes típicos de Cerradão, apresentou baixa similaridade com outras áreas desta fitofisionomia.

Palavras-Chave: caracteres vegetativos, chave dicotômica, ecótono, riqueza de espécies

ABSTRACT - Floristic characterization and dendrological key for species in a Cerradão area in the Cerrado-Pantanal transition, Mato Grosso, Brazil. This study aimed to catalogue and characterize the shrub and tree species in a fragment of Cerradão, in order to produce a dichotomous key based on dendrological characteristics, for the identification of the species in the region. The study area is located in the Cerrado-Pantanal transition, in Cáceres, Mato Grosso State, Brazil, and presents an area of 3 hectares that was divided into 32 plots of $48 \times 20 \mathrm{~m}$. The survey was carried out by inventorying the individuals with a diameter $\geq 5.0 \mathrm{~cm}$, at $30 \mathrm{~cm}$ from the ground. The key was elaborated upon the observed dendrological characters. A total of 84 species, 73 genera and 34 families were recorded. Fabaceae (21 spp.), Malvaceae (6 spp.), Anacardiaceae/Bignoniaceae (5 spp.), Rubiaceae (4 spp.) and Euphorbiaceae/Vochysiaceae ( 3 spp.) accounted for $55.95 \%$ of the species richness. Among the genera, Bauhinia stood out with three species. The studied fragment has a high species richness, and whereas it presents the most typical components of the region, it also presented low similarity with other areas of the same phytophysiognomy.

Keywords: dichotomous key, ecotone, species richness, vegetative features

\section{INTRODUÇÃO}

O Cerrado se estende por 11 estados brasileiros e o Distrito Federal, ocupando cerca de 23,9\% do território nacional (Brasil 2004). Este bioma possui uma flora com extraordinária taxa de endemismo (BFG 2015), e abriga $5 \%$ de toda a diversidade biológica do planeta (Brasil 2010). Além do mais, é considerado um "hotspot" da biodiversidade, sendo uma das áreas de savana mais ricas do mundo (Cavalcanti et al. 2012).

Além da alta riqueza de espécies, a vegetação do Cerrado é constituída por 11 tipologias vegetacionais que variam desde formações florestais, até composições campestres e savânicas, conforme seu aspecto e composição
(Ribeiro \& Walter 2008, Bueno et al. 2018). Neste bioma, o Cerradão se destaca como uma fitofisionomia florestal, composta por espécies de mata de galeria e Cerrado stricto sensu, com aspectos xeromórficos, dossel prevalentemente contínuo, presença de estrato arbustivo e herbáceo, e altura média do estrato arbóreo entre 8 e 15 metros (Ribeiro \& Walter 2008).

Apesar das possibilidades de usos da notável diversidade florística do Cerrado, existe certa carência de ferramentas e de profissionais qualificados para a identificação científica, corroborando para o uso indevido ou a subutilização de seus componentes florestais (Saueressig 2018). Neste cenário, a importância da identificação botânica fundamenta-se em diferentes processos, tanto ecológicos quanto econômicos. 
Estudos florísticos baseados no reconhecimento e classificação botânica podem favorecer a compreensão dos processos ecológicos e colaborar para a predição de quadros futuros da biodiversidade local. Tal fato pode contribuir para geração de informações acerca das variações ocorridas na composição florística após uma supressão pelo fogo, por exemplo (Deus \& Oliveira 2016). Do ponto de vista econômico, a correta identificação de espécies arbóreas é uma das premissas para o manejo florestal sustentável, e configura uma das etapas mais difíceis da exploração sustentável (Alencar 1998), culminando em erros nos inventários florestais. Portanto, no contexto florestal, a identificação incorreta de espécies florestais madeireiras pode, além de outras consequências, apresentar reflexo no processamento da madeira (Procópio \& Secco 2008).

Uma ferramenta valorosa para a correta identificação dos espécimes conforme sua nomenclatura binomial são as chaves dendrológicas, as quais possibilitam a identificação de árvores e arbustos, independente da fenofase (Plazas \& Paula 2016). Desta forma, inúmeros autores vêm desenvolvendo chaves para a identificação dendrológica de diferentes formações vegetais e/ou grupos taxonômicos (Campos Filho \& Sartorelli 2015, Miller \& Blum 2018, Cândido et al. 2019).

Existem chaves dicotômicas consideravelmente extensas e completas baseadas em características reprodutivas, as quais auxiliam a identificação da flora do Cerrado (Zanatta et al. 2015, Souza et al. 2018). Apesar da robustez dessas ferramentas, elas podem ser limitadas, uma vez que, a morfologia dos órgãos reprodutivos nem sempre pode ser avaliada, dada as variações das fenofases. Assim, a confecção de chaves dendrológicas é um processo que permite contornar essas limitações, proporcionando a correta e eficiente identificação dos componentes arbóreos de diferentes fitofisionomias, independente da fenofase, e de considerável importância para a preservação e exploração florestais (Saueressig et al. 2009, Campos Filho \& Sartorelli 2015, Cândido et al. 2019).

O objetivo deste trabalho foi a catalogação e caracterização florística do estrato arbóreo-arbustivo em um fragmento de Cerradão em Cáceres, Mato Grosso, a fim de confeccionar uma chave dicotômica baseada em características dendrológicas para a identificação dos táxons registrados.

\section{MATERIAL E MÉTODOS}

O estudo ocorreu em um fragmento de Cerradão com vegetação secundária de aproximadamente 3 ha localizado na região de transição Cerrado-Pantanal, no Norte do município de Cáceres, Mato Grosso, entre as coordenadas geográficas Latitude Sul 16 07' 53,266" e 16 07' 59,981', e Longitude Oeste $57^{\circ} 41^{\prime}$ 47,806" e 57 41' 41,794', com elevação de 135 m (Fig. 1). A área, denominada de Horto

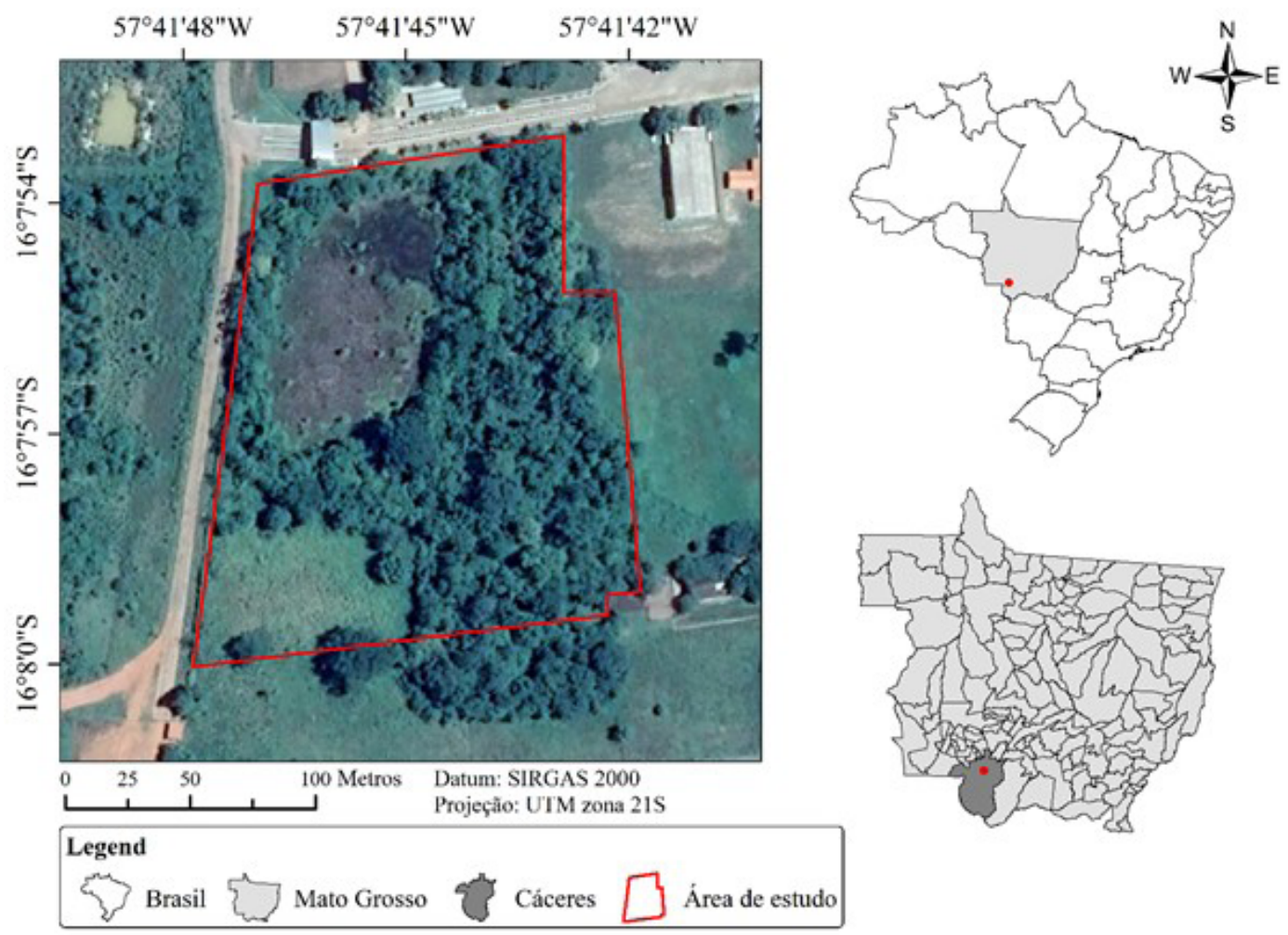

Figura 1. Localização da área de estudo, situada no Horto Florestal Professora Michelle Carmelinda Pegorini Bordini, pertencente ao IFMT Campus Cáceres - Professor Olegário Baldo, Mato Grosso, Brasil. 
Florestal Prof. ${ }^{a}$ Michelle Carmelinda Pegorini Bordini, pertence ao Instituto Federal de Educação Ciência e Tecnologia de Mato Grosso (IFMT) Campus Cáceres - Prof. Olegário Baldo. De acordo com a classificação de Köppen (Köppen \& Geiger 1928), o clima da região é tropical com inverno seco (Awa), apresentando estação chuvosa no verão e inverno seco, com temperatura média anual de $26,24^{\circ} \mathrm{C}$, precipitação total anual de $1335 \mathrm{~mm}$ e umidade relativa do ar média de 78,50\% (Neves et al. 2011).

Aárea foi alvo de uma condição de antropização para uso agrícola e pecuário até 1980 , quando a instituição de ensino foi criada e o local teve o processo de sucessão ecológica iniciado. A vegetação do fragmento é caracterizada como Cerradão (Ribeiro \& Walter 2008). Atualmente é utilizado para práticas de ensino, pesquisa e extensão, desenvolvidas pela comunidade acadêmica e corpo docente do IFMT Campus Cáceres - Prof. Olegário Baldo, o que destaca a importância de se conhecer adequadamente a flora deste ambiente.

O levantamento florístico foi realizado de forma censitária, sendo que a área foi dividida em 32 parcelas de $48 \times 20 \mathrm{~m}$, a fim de facilitar a amostragem dos indivíduos. Foram inventariados todos os fanerófitos arbustivos e arbóreos com diâmetro de $5,0 \mathrm{~cm}$ (DAS $\geq 5,0 \mathrm{~cm}$ ) a $30 \mathrm{~cm}$ do solo, conforme recomendado por Felfili et al. (2011) para estudos florísticos e fitossociológicos em ambientes de Cerrado.

A priori as espécies foram identificadas in loco. Para espécimes não identificados em campo houve registros fotográficos, além de coleta de material botânico, que quando dotados de órgãos reprodutivos foram depositados no Herbário Central da Universidade Federal de Mato Grosso - UFMT (Registros de depósito: Material Suplementar I). Nestes casos, as identificações ocorreram a posteriori, com base em literaturas especializadas (Material Suplementar II), comparações com materiais de referência disponíveis nos portais JBRJ (2020) e CRIA (2020), bem como por consulta a especialistas em identificação botânica.

As espécies foram classificadas de acordo com "Angiosperm Phylogeny Group IV" (APG 2016). A nomenclatura científica foi validada com base na Lista de Espécies da Flora do Brasil (Flora do Brasil 2020, em construção) e pelo Missouri Botanical Garden (Tropicos 2019).

A análise de similaridade florística foi realizada comparando as composições de 11 áreas de Cerradão, disponíveis em dez estudos (Tab. 1). O teste de agrupamento pelo método de Jaccard foi conduzido com base em uma matriz de presença e ausência, sendo que a análise foi processada no software R (R Core Team 2017).

Tabela 1. Relação dos estudos utilizados na análise de similaridade florística.

\begin{tabular}{ll}
\hline Fonte & Local \\
\hline Presente estudo & Cáceres - MT \\
Gomes et al. (2004) & Brotas - SP \\
Marimon-Junior \& Haridasan (2005) & Nova Xavantina - MT \\
Souza et al. (2010) & Paraopeba - MG \\
Camilotti et al. (2011) & Bandeirantes - MS \\
Pinheiro \& Durigan (2012) & Assis-SP \\
Bueno et al. (2013) & Campo Grande - MS \\
Casella \& Silva-Júnior (2013) & Brasília - DF \\
Otoni et al. (2013) & Curvelo - MG \\
Rodrigues \& Araújo (2013) - Fragmento 1 & Uberlândia - MG \\
Rodrigues \& Araújo (2013) - Fragmento 2 & Uberlândia - MG \\
Giácomo et al. (2015) & Morada Nova - MG \\
\hline
\end{tabular}

Para a caracterização dendrológica das espécies foram realizadas observações dos indivíduos em campo e em laboratório, a partir de amostras vegetativas, além da verificação da morfologia em literaturas especializadas. As características vegetativas foram classificadas conforme as terminologias adotadas por Souza \& Lorenzi (2012), Martins-da-Silva et al. (2014) e Saueressig (2017).

A chave dicotômica foi elaborada de modo que os táxons fossem melhor agrupados com base nos seguintes caracteres dendrológicos (Material Suplementar III): hábito (arbustivo, arbóreo e palmeira); tipo de caule (estipe ou tronco); folha (tipo, formato, continuidade do limbo, número de lóbulos, borda, ápice, base, pubescência, pecíolo, nervação, pontuações translúcidas, espinhos, nectários extraflorais, filotaxia e odor); casca externa (aspecto, deiscência, lenticelas, cicatrizes peciolares, acúleos e espinhos); casca interna (cor, textura e exsudação); suberificação do ritidoma; presença de lenticelas e espinhos nos ramos; entre outras. 


\section{RESULTADOS}

Foram catalogadas 84 espécies, distribuídas em 73 gêneros e 34 famílias (Tab. 2). Salienta-se o registo de oito espécies exóticas para a flora brasileira: Mangifera indica L. e Spondias purpurea L. (Anacardiaceae), Albizia lebbeck (L.) Benth. e Leucaena leucocephala (Lam.) de Wit (Fabaceae), Tectona grandis L. f. (Lamiaceae), Psidium guajava L. e Syzygium cumini (L.) Skeels (Myrtaceae), e Citrus $\times$ limon (L.) Osbeck (Rutaceae). Estas espécies correspondem a $9,52 \%$ da riqueza total, enquanto as espécies nativas contabilizaram 76 representantes $(90,48 \%)$.

Tabela 2. Famílias e espécies catalogadas no estrato arbóreo-arbustivo do Horto Florestal Prof. ${ }^{a}$ Michelle Carmelinda Pegorini Bordini, Cáceres-MT e seus respectivos nomes populares (espécies exóticas destacadas com asterisco).

\begin{tabular}{|c|c|}
\hline Famílias/Espécies & Nomes Populares \\
\hline \multicolumn{2}{|l|}{ Anacardiaceae } \\
\hline Anacardium occidentale L. & caju \\
\hline Astronium fraxinifolium Schott & gonçaleiro \\
\hline Astronium urundeuva (M. Allemão) Engl. & aroeira \\
\hline Mangifera indica $\mathrm{L}$. & manga* \\
\hline Spondias purpurea $\mathrm{L}$. & seriguela* \\
\hline \multicolumn{2}{|l|}{ Annonaceae } \\
\hline Annona cornifolia A.St.-Hil. & ata-do-campo \\
\hline \multicolumn{2}{|l|}{ Apocynaceae } \\
\hline Aspidosperma cylindrocarpon Müll.Arg. & peroba-rosa \\
\hline Aspidosperma subincanum Mart. & guatambu \\
\hline \multicolumn{2}{|l|}{ Arecaceae } \\
\hline Acrocomia aculeata (Jacq.) Lodd. ex Mart & bocaiúva \\
\hline \multicolumn{2}{|l|}{ Bignoniaceae } \\
\hline Cybistax antisyphilitica (Mart.) Mart. & ipê-verde \\
\hline Handroanthus chrysotrichus (Mart. ex DC.) Mattos & ipê-amarelo \\
\hline Handroanthus impetiginosus (Mart. ex DC.) Mattos & ipê-roxo \\
\hline Tabebuia aurea (Silva Manso) Benth. \& Hook.f. ex S.Moore & para-tudo \\
\hline Tabebuia roseoalba (Ridl.) Sandwith & ipê-branco \\
\hline \multicolumn{2}{|l|}{ Bixaceae } \\
\hline Bixa orellana $\mathrm{L}$. & urucum \\
\hline \multicolumn{2}{|l|}{ Boraginaceae } \\
\hline Cordia glabrata (Mart.) A.DC. & louro-preto \\
\hline Cordia trichotoma (Vell.) Arráb. ex Steud. & louro-pardo \\
\hline \multicolumn{2}{|l|}{ Burseraceae } \\
\hline Protium heptaphyllum (Aubl.) Marchand & breu-branco \\
\hline \multicolumn{2}{|l|}{ Cannabaceae } \\
\hline Celtis iguanaea (Jacq.) Sarg. & grão-de-galo \\
\hline Trema micrantha $(\mathrm{L}$.$) Blume$ & periquiteira \\
\hline \multicolumn{2}{|l|}{ Celastraceae } \\
\hline Monteverdia gonoclada (Mart.) Biral & coração-de-bugre \\
\hline \multicolumn{2}{|l|}{ Dilleniaceae } \\
\hline Curatella americana $\mathrm{L}$. & lixeira \\
\hline \multicolumn{2}{|l|}{ Ebenaceae } \\
\hline Diospyros inconstans Jacq. & marmelinho \\
\hline \multicolumn{2}{|l|}{ Erythroxylaceae } \\
\hline Erythroxylum anguifugum Mart. & pimenteirinha \\
\hline Erythroxylum deciduum A.St.-Hil. & ata-de-cobra \\
\hline \multicolumn{2}{|l|}{ Euphorbiaceae } \\
\hline Alchornea discolor Poepp. & supiarana \\
\hline
\end{tabular}


Tabela 2. Cont.

Famílias/Espécies

Cnidoscolus urens (L.) Arthur

Manihot anomala Pohl

Fabaceae

Albizia lebbeck (L.) Benth.

Anadenanthera colubrina (Vell.) Brenan

Bauhinia pentandra (Bong.) D.Dietr.

Bauhinia rufa (Bong.) Steud.

Bauhinia ungulata $\mathrm{L}$.

Cenostigma bracteosum (Tul.) E. Gagnon \& G.P. Lewis

Dipteryx alata Vogel

Diptychandra aurantiaca Tul.

Enterolobium contortisiliquum (Vell.) Morong

Hymenaea martiana Hayne

Inga laurina (Sw.) Willd.

Leucaena leucocephala (Lam.) de Wit

Luetzelburgia praecox (Harms) Harms

Machaerium acutifolium Vogel

Machaerium villosum Vogel

Platypodium elegans Vogel

Pterogyne nitens Tul.

Schizolobium parahyba (Vell.) Blake

Senegalia tenuifolia (L.) Britton \& Rose

Swartzia jorori Harms

Vatairea macrocarpa (Benth.) Ducke

Lamiaceae

Tectona grandis L. f.

Vitex cymosa Bertero ex Spreng.

Lythraceae

Physocalymma scaberrimum Pohl

Malpighiaceae

Bunchosia paraguariensis Nied.

Malvaceae

Guazuma ulmifolia Lam.

Helicteres lhotzkyana (Schott \& Endl.) K.Schum.

Luehea grandiflora Mart. \& Zucc.

Luehea paniculata Mart. \& Zucc.

Pseudobombax tomentosum (Mart.) A.Robyns

Sterculia striata A.St.-Hil. \& Naudin

Meliaceae

Cedrela fissilis Vell.

Moraceae

Maclura tinctoria (L.) D.Don ex Steud.

Myrtaceae

Psidium guajava $\mathrm{L}$.

Syzygium cumini (L.) Skeels

Nyctaginaceae

Neea hermaphrodita S.Moore

Oleaceae
Nomes Populares

cansanção

mandioca-de-veado

$$
\begin{gathered}
\text { faveiro* } \\
\text { angico } \\
\text { capa-bode } \\
\text { pata-de-vaca } \\
\text { pata-de-vaca } \\
\text { catingueira } \\
\text { cumbaru } \\
\text { carvão-vermelho } \\
\text { tamboril } \\
\text { jatobá } \\
\text { ingá-mirim } \\
\text { leucena* } \\
\text { pau-mocó } \\
\text { jacarandá-do-campo } \\
\text { jacarandá-do-cerrado } \\
\text { amendoim-do-campo } \\
\text { amendoim-bravo } \\
\text { pinho-cuiabano } \\
\text { unha-de-gato } \\
\text { justa-conta } \\
\text { angelim-do-cerrado }
\end{gathered}
$$

teca*

tarumã

quebra-machado

chico-magro

saca-rolha

açoita-cavalo

açoita-cavalo

embiruçu

chichá

cedro-rosa

amoreira-do-campo

goiaba*

jamelão*

pau-de-sal 
Tabela 2. Cont.

\begin{tabular}{|c|c|}
\hline Famílias/Espécies & Nomes Populares \\
\hline Priogymnanthus hasslerianus (Chodat) P.S.Green & osso-de-burro \\
\hline \multicolumn{2}{|l|}{ Piperaceae } \\
\hline Piper arboreum Aubl. & falso-jaborandi \\
\hline \multicolumn{2}{|l|}{ Rhamnaceae } \\
\hline Rhamnidium elaeocarpum Reissek & cabriteiro \\
\hline \multicolumn{2}{|l|}{ Rubiaceae } \\
\hline Chomelia pohliana Müll.Arg. & espinheiro-do-mato \\
\hline Cordiera sessilis (Vell.) Kuntze & marmelada \\
\hline Genipa americana $\mathrm{L}$. & jenipapo \\
\hline Tocoyena formosa (Cham. \& Schltdl.) K.Schum. & jenipapo-de-cavalo \\
\hline \multicolumn{2}{|l|}{ Rutaceae } \\
\hline Citrus $\times$ limon $($ L.) Osbeck & limão* \\
\hline Zanthoxylum riedelianum Engl. & mamica-de-porca \\
\hline \multicolumn{2}{|l|}{ Salicaceae } \\
\hline Banara arguta Briq. & sardinheira \\
\hline Casearia sylvestris $\mathrm{Sw}$. & guaçatonga \\
\hline \multicolumn{2}{|l|}{ Sapindaceae } \\
\hline Dilodendron bipinnatum Radlk. & maria-pobre \\
\hline Magonia pubescens A.St.-Hil. & timbó \\
\hline \multicolumn{2}{|l|}{ Sapotaceae } \\
\hline Pouteria gardneri (Mart. \& Miq.) Baehni & sapotinha \\
\hline Pouteria glomerata (Miq.) Radlk. & laranjinha-de-pacú \\
\hline \multicolumn{2}{|l|}{ Solanaceae } \\
\hline Solanum acanthodes Hook.f. & jurubebão \\
\hline \multicolumn{2}{|l|}{ Urticaceae } \\
\hline Cecropia pachystachya Trécul & embaúba \\
\hline \multicolumn{2}{|l|}{ Vochysiaceae } \\
\hline Callisthene fasciculata Mart. & carvão-branco \\
\hline Qualea grandiflora Mart. & pau-terra-do-cerrado \\
\hline Vochysia divergens Pohl & cambará \\
\hline
\end{tabular}

Em relação aos gêneros, Bauhinia L. foi o único que apresentou três táxons, enquanto que para Aspidosperma Mart. \& Zucc., Astronium Jacq., Cordia L., Erythroxylum P.Browne, Handroanthus Mattos, Luehea Willd., Machaerium Pers., Pouteria Aubl. e Tabebuia Gomes ex DC., foram amostradas duas espécies. As famílias com maior riqueza específica foram Fabaceae (21), seguida por Malvaceae (seis), Anacardiaceae e Bignoniaceae (cinco cada), Rubiaceae (quatro cada), e Euphorbiaceae e Vochysiaceae (três cada), que contabilizaram 55,95\% da riqueza total.

O levantamento do estrato arbóreo-arbustivo das 11 áreas analisadas para similaridade florística e do presente trabalho totalizou 360 espécies. A análise de agrupamento demonstrou que a área do presente estudo apresenta baixa similaridade em relação às demais áreas de Cerradão comparadas. Esta situação é evidenciada pelo isolamento do nosso estudo no dendrograma de similaridade, resultante do teste de agrupamento (Fig. 2).

A chave dicotômica confeccionada utilizando-se exclusivamente características vegetativas é composta por 83 passos, nos quais é possível identificar as espécies arbóreo-arbustivas e a espécie de palmeira levantadas no Horto Florestal Prof. ${ }^{a}$ Michelle Carmelinda Pegorini Bordini. As fotografias das espécies estão disponibilizadas no Material Suplementar IV. 


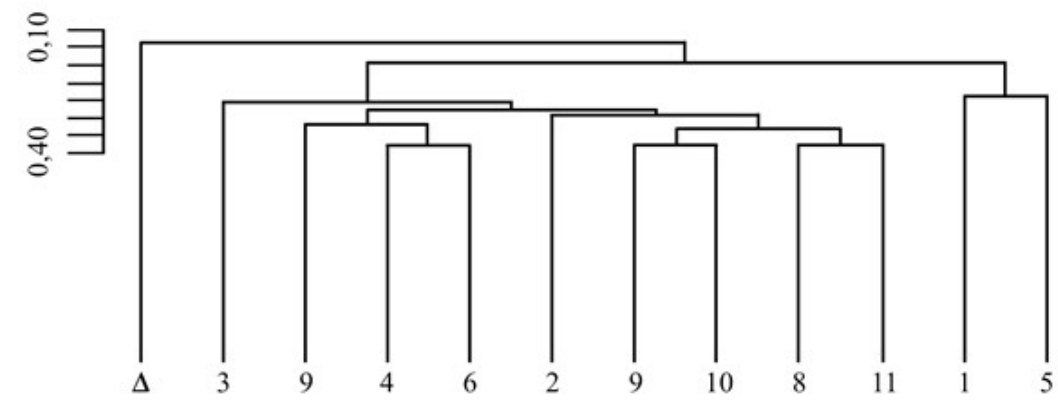

Figura 2. Dendrograma de agrupamento realizado pelo método de Jaccard da similaridade florística do fragmento estudado e outros estudos em área de Cerradão. $\Delta$. Presente Estudo; 1. Gomes et al. (2004); 2. Marimon-Junior \& Haridasan (2005); 3. Souza et al. (2011); 4. Camilotti et al. (2011); 5. Pinheiro \& Durigan (2012); 6. Bueno et al. (2013); 7. Casella \& Silva-Júnior (2013); 8. Otoni et al. (2013); 9. Rodrigues \& Araújo (2013) - Fragmento 1; 10. Rodrigues \& Araújo (2013) - Fragmento 2; 11. Giácomo et al. (2015).

\section{Chave para identificação das espécies arbóreo-arbustivas do Horto Florestal Prof. a Michelle Carmelinda Pegorini Bordini, Cáceres, Mato Grosso, Brasil}

1. Caule do tipo estipe Acrocomia aculeata

1'. Caule do tipo tronco

2. Folha simples

\section{Limbo fendido}

4. Folha peltada, discolor, face abaxial acinzentada, presença de lenticelas e cicatrizes peciolares no tronco e galhos Cecropia pachystachya

4'. Folha não peltada

5. Exsudação ausente e hábito predominantemente arbóreo

6. Folha pinatífida, filotaxia alterna espiralada, porte arbóreo ou arbustivo, casca externa finamente estriada, esparsamente aculeada Solanum acanthodes

6'. Folha palmatífida, 3-5-lobada, filotaxia alterna espiralada, porte arbóreo, casca externa áspera, lenticelada Sterculia striata

5'. Exsudação presente e hábito arbustivo

7. Folha palmatífida, 3-5-lobada com presença de tricomas urticantes na lâmina foliar, pecíolo, ramos, inflorescências e frutos Cnidoscolus urens

7'. Folha geralmente 3-lobada, podendo apresentar heterofilia com limbo 2-lobado ou, menos frequentemente, contínuo

Manihot anomala

3’. Limbo não fendido

8. Filotaxia oposta ou verticilada

9. Folhas verticiladas ou predominantemente verticiladas

10. Hábito arbustivo, casca externa áspera, enegrecida e lenticelada, filotaxia variável, apresentando folhas arranjadas de maneira alterna, oposta, suboposta, ou mais frequentemente, (3-)4-5-verticilada

Neea hermaphrodita

10'. Hábito arbóreo, casca externa fissurada de cor branco acinzentada, disposição foliar padronizada, folhas 3-4-verticiladas Vochysia divergens

9’. Folhas opostas dísticas ou opostas cruzadas

11.Filotaxia oposta dística

12. Ritidoma com pouca ou nenhuma suberificação Bunchosia paraguariensis

12'. Ritidoma muito suberificado 
13. Casca interna bege ou branco-amarelada Callisthene fasciculata

13'. Casca interna rosa-avermelhada

14. Textura da casca interna arenosa Physocalymma scaberrimum

14'. Textura da casca interna fibrosa Rhamnidium elaeocarpum

11'. Filotaxia oposta cruzada

15. Hábito arbustivo

16. Ramos espinescentes, espinhos axilares eretos Chomelia pohliana

16'. Ramos inermes

17. Casca externa fissurada, lenticelas ausentes, desprendimento em tiras, folhas glabras .... Cordiera sessilis

17'. Casca externa áspera, lenticelas presentes, desprendimento pulverulento, folhas pubescentes Tocoyena formosa

15’. Hábito arbóreo

18. Ritidoma suberificado

19. Casca interna marrom escuro Qualea grandiflora

19'. Casca interna branco-amarelada Tectona grandis

18 '. Ritidoma não suberificado

20. Casca externa lisa, desprendimento em lâminas Psidium guajava

20 . Casca externa áspera, raramente rugosa

21. Folhas com pontuações translúcidas Syzygium cumini 21'. Folhas sem pontuações translúcidas

22. Cicatrizes peciolares e lenticelas presentes Genipa americana 22'. Cicatrizes peciolares e lenticelas ausentes Priogymnanthus hasslerianus

8'. Filotaxia alterna

\section{Filotaxia alterna dística}

24. Borda do limbo inteira

25. Ritidoma não suberificado, casca externa áspera, cicatrizes peciolares e lenticelas presentes, base foliar assimétrica, pecíolo glanduloso Piper arboreum

25’. Ritidoma com alguma suberificação

26. Limbo foliar pubescente Annona cornifolia

26'. Limbo foliar glabrescente Diospyros inconstans

24'. Borda do limbo de outros tipos, mas não inteira

27. Ritidoma com desprendimento pulverulento

28. Exsudação presente Maclura tinctoria

28'. Exsudação ausente

29. Ramos espinescentes Celtis iguanaea

29'. Ramos inermes

30. Hábito arbustivo Helicteres lhotzkyana

30’. Hábito arbóreo 
31. Limbo orbicular a largamente oblongo, marcadamente discolor, face abaxial verdeacinzentada, base fortemente cordada Luehea grandiflora

31'. Limbo lanceolado, ligeiramente discolor, base obtusa, levemente assimétrica ou ligeiramente cordada Trema micrantha

27'. Ritidoma com outras formas de desprendimento

32. Casca interna bege

33. Nectários extraflorais presentes no ápice do pecíolo Banara arguta

33'. Nectários extraflorais ausentes Casearia sylvestris

32'. Casca interna rosa-avermelhada

34. Base da folha aguda ou cuneada Monteverdia gonoclada

34'. Base da folha cordada

35. Ritidoma com desprendimento em escamas Guazuma ulmifolia

35 . Ritidoma com desprendimento em pequenas placas retangulares

Luehea paniculata

23'. Filotaxia alterna espiralada

36. Planta com odor de terebintina

37. Limbo com formato obovado Anacardium occidentale

37'. Limbo com formato lanceolado Mangifera indica

36'. Planta sem odor de terebintina

38. Exsudação ausente

39. Casca interna bege ou branco-amarelada

40. Ritidoma com pouca ou nenhuma suberificação

41. Venação peninérvia, limbo elíptico a oblongo, menos comumente ovalado, base arredondada ou obtusa, borda crenada Alchornea discolor

41'. Venação palminérvia, limbo deltoide a cordado, menos comumente ovalado, base truncada ou cordada, borda inteira Bixa orellana

40 '. Ritidoma muito suberificado

42. Casca externa reticulada Cordia glabrata

42 '. Casca externa fissurada Cordia trichotoma

39'. Casca interna rosa-avermelhada

43. Casca externa rugosa Curatella americana

43'. Casca externa fissurada ou reticulada

44. Ápice agudo a raramente arredondado, base arredondada a cuneada Erythroxylum anguifugum

44'. Ápice levemente emarginado a mucronado, base aguda a cuneada

Erythroxylum deciduum

38'. Exsudação presente

45. Ramos não lenticelados Pouteria glomerata

45'. Ramos lenticelados

46. Ritidoma não suberificado, casca externa áspera, densamente lenticelada, folhas congestas nas extremidades dos ramos Aspidosperma subincanum

46'. Ritidoma suberificado, casca externa fissurada ou rugosa, não lenticelada, folhas não congestas nas extremidades dos ramos 
47. Folhas com face abaxial verde claro, venação broquidódroma Aspidosperma cylindrocarpon

47'. Folhas com face abaxial glauca, venação eucamptódroma Pouteria gardneri

2'. Folha composta

48. Folha unifoliolada, com pontuações translúcidas e pecíolo ligeiramente alado Citrus $\times$ limon

48'. Outros tipos de folha composta

49. Folha trifoliolada Tabebuia roseoalba

49’. Folha bifoliolada, digitada, pinada ou paribipinada

50. Folha bifoliolada

51. Folíolos não concrescidos Hymenaea martiana

51'. Folíolos concrescidos

52. Casca externa lenticelada, áspera, desprendimento pulverulento Bauhinia pentandra

$52^{\prime}$. Casca externa não lenticelada, fissurada ou reticulada

53. Casca externa fissurada, casca interna rosa-avermelhada, nervuras terciárias proeminentes na face abaxial Bauhinia rufa

53'. Casca externa finamente reticulada, casca interna bege, nervuras terciárias impressas em ambas as faces

Bauhinia ungulata

50 . Folha digitada, pinada ou paribipinada

54. Folha digitada

55. Ritidoma com pouca ou nenhuma suberificação

56. Casca externa superficialmente fissurada, casca interna bege, base dos folíolos arredondada ou obtusa Handroanthus impetiginosus

56. Casca externa superficialmente reticulada, casca interna branco-amarelada, base dos folíolos aguda ou cuneada Vitex cymosa

55'. Ritidoma com muita suberificação

57. Filotaxia alterna espiralada, casca externa fissurada, presença de fissuras verdes, folíolos sésseis e discolores Pseudobombax tomentosum

57'. Filotaxia oposta cruzada, casca externa fissurada, fissuras verdes ausentes

58. Folhas geralmente 5 -folioladas, folíolos pubescentes, tricomas estrelados Handroanthus chrysotrichus

58'. Folhas geralmente 7-folioladas, folíolos glabros

59. Base dos folíolos decurrente, ápice acuminado Cybistax antisyphilitica

59’. Base dos folíolos arredondada, ápice arredondado ou obtuso .... Tabebuia aurea

54'. Folha pinada ou paribipinada

60. Folha pinada

61. Folha paripinada

62. Filotaxia alterna dística Platypodium elegans

62 '. Filotaxia alterna espiralada

63. Casca externa com acúleos Zanthoxylum riedelianum

63’. Casca externa sem acúleos 
64. Ritidoma não suberificado, casca externa áspera, lenticelas presentes, folhas com nectários extraflorais Inga laurina

64'. Ritidoma suberificado, casca externa fissurada ou rugosa, lenticelas ausentes, folhas sem nectários extraflorais

65. Casca externa fissurada Cedrela fissilis

\section{5'. Casca externa rugosa}

66. Casca interna intensamente rosa-avermelhada ... Diptychandra aurantiaca

66'. Casca interna marrom ou suavemente rosa-avermelhada

67. Folha com raque alada

Dipteryx alata

67 '. Folha com raque não alada

Magonia pubescens

61'. Folha imparipinada

\section{Casca externa rugosa}

69. Folíolos com ápice acuminado, base truncada a levemente cordada, desprendimento em escamas formando depressões discolores Astronium fraxinifolium

69'. Folíolos com ápice cuneado, base arredondada, desprendimento em placas sem formar depressões descolores Astronium urundeuva

68'. Casca externa estriada, fissurada ou reticulada

70. Filotaxia alterna dística Swartzia jorori

70'. Filotaxia alterna espiralada

71. Planta com odor de terebintina

72. Casca externa reticulada, folha 7-foliolada, raramente 5-foliolada, margem dos folíolos inteira Protium heptaphyllum

72'. Casca externa estriada nos galhos e troncos jovens e grossamente rugosa nos galhos e troncos maduros, folha $>7$-foliolada, margem dos folíolos suavemente crenada Spondias purpurea

71'. Planta sem odor de terebintina

73. Ritidoma com suberificação reduzida

74. Margem dos folíolos suavemente crenada Luetzelburgia praecox

74'. Margem dos folíolos inteira Pterogyne nitens

73'. Ritidoma com suberificação proeminente

75. Ramos não lenticelados Vatairea macrocarpa

75'. Ramos lenticelados

76. Limbo foliar glabro Machaerium acutifolium

76'. Limbo foliar velutino Machaerium villosum

60'. Folha paribipinada

77. Casca externa lenticelada, aparência áspera ou estriada

78. Casca externa com cicatrizes peciolares Schizolobium parahyba

78'. Casca externa sem cicatrizes peciolares

79. Casca interna intensamente rosa-avermelhada Albizia lebbeck

79'. Casca interna bege ou branco-amarelada

80. Hábito arbustivo, tronco geralmente irregular Cenostigma bracteosum 80 '. Hábito arbóreo, troco cilíndrico 
81. Casca interna bege, foliólulos lanceolados Enterolobium contortisiliquum

81'. Casca interna branco-amarelada, foliólulos geralmente oblongos Leucaena leucocephala

77’. Casca externa não lenticelada, aparência fissurada ou rugosa

82. Nectários extraflorais ausentes, borda dos foliólulos serreada, discolores, casca externa rugosa, casca interna intenso-avermelhada Dilodendron bipinnatum

82'. Nectários extraflorais presentes, borda dos foliólulos inteira

83. Casca externa rugosa, ramos e folhas inermes, nectários extraflorais avermelhados entre os últimos pares de pinas Anadenanthera colubrina

83'. Casca externa fissurada, ramos e folhas armados, espinhos recurvados na face abaxial da raque Senegalia tenuifolia

\section{DISCUSSÃO}

A maior expressividade de Fabaceae na comunidade estudada é justificada pela abrangência e riqueza desta família na flora brasileira (Souza et al. 2018) e por ser a terceira família botânica mais numerosa em termos de espécies no mundo (Lewis et al. 2005, LPWG 2013). Sua tendência cosmopolita proporciona a maior riqueza do grupo no estrato arbóreo-arbustivo de diversas fitofisionomias do Cerrado, como no Cerrado stricto sensu (Silva et al. 2016, Gama et al. 2018), Matas de Galeria (Matos \& Felfili 2010, Loschi et al. 2013), e em campo sujo (Giácomo et al. 2013). Em ambiente de Cerradão, diversos estudos revelam a ocorrência de uma ampla diversidade de táxons desta família (Gomes et al. 2004, Bueno et al. 2013, Otoni et al. 2013, Giácomo et al. 2015).

Boa parte das espécies de Fabaceae que ocorrem no Cerrado, englobando as arbóreas, são capazes de fixar Nitrogênio por meio de associações simbióticas (Bustamante et al. 2004, 2012). A capacidade de fixação biológica de Nitrogênio confere vantagens competitivas nas diferentes fitofisionomias do bioma, culminando em alto número de táxons desta família nestes ambientes (Gama et al. 2018). A grande diversidade desta família na área de estudo pode ser creditada, ainda, ao estágio sucessional do fragmento, uma vez que estudos revelam a predominância de espécies do grupo em áreas com vegetação resultante do processo de sucessão secundária (Deus \& Oliveira 2016, Sabino et al. 2016). Além de Fabaceae, famílias como Anacardiaceae, Bignoniaceae, Euphorbiaceae, Malvaceae, Rubiaceae e Vochysiaceae também apresentaram considerável riqueza de espécies em diferentes fitofisionomias do Cerrado, conforme relacionado na Tabela 3.

Por outro lado, Myrtaceae, que é uma família amplamente registrada entre as de maior riqueza em ambiente de Cerradão (Gomes et al. 2004, Camilotti et al. 2011, Casella \& Silva Júnior 2013), não foi representada por táxons nativos na área estudada, sendo observadas apenas as espécies exóticas Psidium guajava e Syzygium cumini.
Grande parte dos gêneros com duas ou mais espécies no fragmento estudado também foram amostrados entre os mais importantes em outros estudos no Cerrado: Bauhinia (Siqueira et al. 2006), Aspidosperma (Stefanello et al. 2009, Cândido et al. 2018), Astronium Jacq. (Souza et al. 2010, Maria et al. 2018), Erythroxylum (Siqueira et al. 2006, Bueno et al. 2013, Casella \& Silva Júnior 2013, Otoni et al. 2013), Handroanthus (Matos \& Felfili 2010, Casella \& Silva Júnior 2013, Otoni et al. 2013), Machaerium (Loschi et al. 2013, Cândido et al. 2018), e Pouteria (Stefanello et al. 2009). Por outro lado, Qualea Aubl., que comumente é registrado entre os gêneros mais ricos no Cerrado (Siqueira et al. 2006, Casella \& Silva Júnior 2013, Cândido et al. 2018, Gama et al. 2018), foi representado apenas por Qualea grandiflora.

A composição florística do Cerradão estudado se sobrepõe a de outras tipologias vegetacionais do bioma Cerrado. Por exemplo, espécies como Cordia trichotoma, Enterolobium contortisiliquum, Guazuma ulmifolia, Physocalymma scaberrium, Tabebuia aurea (Oliveira et al. 2018), Astronium fraxinifolium, Protium heptaphyllum e Qualea grandiflora (Gama et al. 2018), entre outras, reportadas em Cerrado stricto sensu; Anacardium occidentale, Callisthene fasciculata, Luetzelburgia praecox, Pseudobombax tomentosum, Tocoyena formosa, entre outras, em cerrado rupestre (Gomes et al. 2011); e Annona cornifolia, Aspidosperma subincanum, Diptychandra aurantiaca, Luehea grandiflora, Pouteria gardneri, entre outras, em mata de galeria (Cabacinha \& Fontes 2014).

O compartilhamento de espécies desta vegetação apresenta-se ainda mais extenso, visto a existência de espécies que possuem ocorrência abrangente no território nacional. Por exemplo, Anadenanthera colubrina, Casearia sylvestris, Cecropia pachystachya, Guazuma ulmifolia, Handroanthus impetiginosus, Maclura tinctoria, Astronium urundeuva, Pterogyne nitens, Rhamnidium elaeocarpum e Trema micrantha, espécies encontradas neste trabalho, e que detêm ampla distribuição em diferentes formações vegetacionais do Cerrado (Maria et al. 2018). 
Tabela 3. Relação de pesquisas realizadas no estrato arbóreo-arbustivo de diferentes fitofisionomias do Cerrado, apontando as famílias com maior riqueza de espécies (exceto Fabaceae).

\begin{tabular}{|c|c|c|c|}
\hline Família & Fitofisionomia & Fonte & Número de Espécies \\
\hline \multirow{4}{*}{ Anacardiaceae } & Mata de galeria & Matos \& Felfili (2010) & 4 \\
\hline & Cerradão & Bueno et al. (2013) & 3 \\
\hline & Cerradão & Otoni et al. (2013) & 3 \\
\hline & Stricto sensu & Cândido et al. (2018) & 4 \\
\hline \multirow{5}{*}{ Bignoniaceae } & Cerradão & Camilotti et al. (2011) & 3 \\
\hline & Mata seca & Giácomo et al. (2015) & 3 \\
\hline & Cerradão & Giácomo et al. (2015) & 3 \\
\hline & Stricto sensu & Silva et al. (2016) & 6 \\
\hline & Stricto sensu & Cândido et al. (2018) & 3 \\
\hline \multirow{2}{*}{ Euphorbiaceae } & Cerradão & Gomes et al. (2004) & 5 \\
\hline & Stricto sensu & Oliveira et al. (2018) & 4 \\
\hline \multirow{4}{*}{ Malvaceae } & Cerradão & Camilotti et al. (2011) & 3 \\
\hline & Mata seca & Giácomo et al. (2015) & 4 \\
\hline & Stricto sensu & Cândido et al. (2018) & 4 \\
\hline & Stricto sensu & Oliveira et al. (2018) & 6 \\
\hline \multirow{5}{*}{ Rubiaceae } & Cerradão & Gomes et al. (2004) & 7 \\
\hline & Cerradão & Camilotti et al. (2011) & 7 \\
\hline & Cerradão & Casella \& Silva Júnior (2013) & 5 \\
\hline & Cerradão & Otoni et al. (2013) & 8 \\
\hline & Mata seca & Giácomo et al. (2015) & 3 \\
\hline \multirow{5}{*}{ Vochysiaceae } & Cerradão & Camilotti et al. (2011) & 9 \\
\hline & Cerradão & Bueno et al. (2013) & 6 \\
\hline & Cerradão & Casella \& Silva Júnior (2013) & 6 \\
\hline & Campo sujo & Giácomo et al. (2013) & 5 \\
\hline & Stricto sensu & Silva et al. (2016) & 6 \\
\hline
\end{tabular}

Mesmo compartilhando espécies de ampla ocorrência, a análise de similaridade florística apontou que o fragmento estudado apresenta composição diferente das demais áreas de Cerradão comparadas. Por estar em área de transição, o fragmento ostenta espécies de diferentes ecossistemas, e por isso sua florística é pouco similar às demais formações de mesma fitofisionomia, demonstrando a importância desse tipo de fragmento para conservação da biodiversidade na região. A dissimilaridade observada corrobora os apontamentos de Milan \& Moro (2016), os quais afirmam que a riqueza em áreas de ecótono é elevada, e apresenta espécies típicas de ambos os Biomas além de registros de táxons que possuem sua distribuição relacionada com as zonas de transição.

Estudos que abrangem regiões transicionais entre biomas apontam a composição florística singular destas áreas (Marimon et al. 2014). Assim, é comum que os fragmentos existentes em regiões ecotonais apresentem baixa similaridade com outras formações. Esse fenômeno é verificado ao se observar o dendrograma de similaridade, onde o presente trabalho e os estudos de Gomes et al. (2004) e Pinheiro \& Durigan (2012) foram fortemente dissimilares das demais áreas avaliadas, sendo estes últimos, levantamentos realizados em áreas de transição no estado de São Paulo.

A transição entre diferentes biomas e seus domínios não ocorre de forma abrupta, fazendo com que existam áreas meandrantes, com encraves do Pantanal no Cerrado e vice- -versa, havendo regiões que são consideradas como faixas transicionais (Marques et al. 2020). Nesta perspectiva, estudos realizados no Pantanal têm reportado a ocorrência de inúmeras espécies amplamente distribuídas no Cerrado, e também amostradas no presente levantamento, tais como Aspidosperma subincanum, Astronium fraxinifolium, Casearia sylvestris, Cordia glabrata, Dilodendron bipinnatum, Dipteryx alata, Protium heptaphyllum, Luehea paniculata, Magonia pubescens, Astronium urundeuva, Qualea grandiflora, Tocoyena formosa e Vatairea macrocarpa, entre outras (Costa et al. 2010, Morais et al. 2013).

O fragmento ostenta espécies notadamente pertencentes à flora pantaneira, reforçando a condição ecotonal da área, tais como Alchornea discolor, Banara arguta (Castrillon et al. 2011, Martins et al. 2020), Bunchosia paraguariensis (Flora do Brasil 2020, em construção), Erythroxylum anguifugum, Neea hermaphrodita (Morais et al. 2013), Pouteria glomerata (Martins et al. 2020) e Swartzia jorori (Castrillon et al. 2011). Em alguns casos, espécies reconhecidas por comporem vastas paisagens com populações monodominantes no Pantanal, a exemplo dos paratudais, formados por Tabebuia aurea (Pott et al. 2011) e os cambarazais, que reúnem centenas de indivíduos de Vochysia divergens (Pott et al. 2011, Morais et al. 2013). Neste contexto, Solórzano et al. (2012) apontam para a propensão das formações de Cerradão em assimilarem componentes florísticos das fisionomias vegetacionais adjacentes. 
Algumas espécies encontradas no fragmento são ameaçadas de extinção em nível global, na categoria vulnerável (VU), de acordo com IUCN (2018): Dipteryx alata, Machaerium villosum e Cedrela fissilis. A existência destas espécies na área ressalta a importância de preservação de fragmentos florestais, sobretudo em áreas de transição, as quais geralmente abrigam grande biodiversidade e sofrem maior tensão ecológica (Marques et al. 2020).

Em relação aos caracteres utilizados na distinção das espécies, a diferenciação do tipo de caule em tronco e estipe foi suficiente para distinguir a espécie de palmeira (Acrocomia aculeata) e as espécies arbóreas e arbustivas. Para estas espécies, características foliares como tipo, borda e filotaxia foram fundamentais para a distinção de grandes grupos de plantas. Já fatores como o hábito, aspecto da casca externa, exsudação, intensidade de suberificação e presença de espinhos foram adequados para a segregação das espécies em grupos menores. A identificação em nível específico se deu por meio de caracteres menos abrangentes como nectários extraflorais, pontuações translúcidas, venação, presença de lenticelas e cor da casca interna. Miller \& Blum (2018) e Cândido et al. (2019) também empregaram com sucesso o tipo de folha e características do ritidoma na elaboração de chaves dendrológicas.

A caracterização e classificação da macromorfologia vegetativa de espécies lenhosas tem sido utilizada com sucesso na identificação dendrológica de grupos taxonômicos extensos, como é o caso da família Fabaceae (Cândido et al. 2019). Ademais, chaves de identificação dendrológica são eficazes no reconhecimento rápido de essências florestais no Cerrado, colaborando na correta identificação de táxons ao longo de vegetações semelhantes, além de permitirem que novos estudos sejam realizados, fortalecendo as práticas científicas e acadêmicas (Miller \& Blum 2018). A utilização destas ferramentas é importante no reconhecimento de táxons potenciais para a restauração florestal, determinando grupos de espécies prioritárias na recuperação de áreas degradadas (Campos Filho \& Sartorelli 2015).

O fragmento possui alta riqueza ( 84 espécies, 73 gêneros e 34 famílias), sendo a maior parte dos componentes típicos de Cerradão e alguns táxons típicos da flora do Pantanal, além de oito componentes exóticos. Fabaceae foi a família com maior riqueza, sendo Bauhinia o gênero com maior número de espécies. O padrão de riqueza observado é semelhante aos de outros fragmentos de Cerradão. A composição florística do fragmento estudado não foi similar a nenhum dos estudos analisados, correspondendo a padrões de similaridade observados em outras áreas de Cerradão em zona de transição. Os caracteres dendrológicos utilizados foram adequados na construção da chave dicotômica, resultando em uma ferramenta eficiente para a identificação de espécies florestais, e facilitará o trabalho dos profissionais da área.

\section{AGRADECIMENTOS}

Ao CNPq pela concessão de Bolsa de Iniciação científica ao primeiro autor, através do financiamento do projeto NEURODENDRO - Sistema Inteligente de Identificação de Espécies Florestais do Pantanal Matogrossense. Ao dendrólogo Libério Amorim, pelas valiosas colaborações na identificação das espécies. Ao curso de Engenharia Florestal do IFMT Campus Cáceres - Prof. Olegário Baldo, sobretudo aos colaboradores do laboratório de Biologia Vegetal e Dendrologia.

\section{MATERIAL SUPLEMENTAR}

O seguinte material online está disponível para este artigo: I. Registros de tombamento das exsicatas elaboradas; II. Literatura auxiliar utilizada para identificação das espécies; III. Glossário; IV. Pranchas com material fotográfico das espécies levantadas. Disponível em: https://isb.emnuvens. com.br/iheringia/article/view/934/527.

\section{REFERÊNCIAS}

Alencar, J. C. 1998. Identificação Botânica de Árvores de Floresta Tropical Úmida da Amazônia por meio de Computador. Acta Amazonica 28(1):3-30.

APG IV (The Angiosperm Phylogeny Group). 2016. An update of the Angiosperm Phylogeny Group classification for the orders and families of flowering: APG IV. Botanical Journal of the Linnean Society 181(1):1-20.

BFG (The Brazil Flora Group). 2015. Growing knowledge: an overview of Seed Plant diversity in Brazil. Rodriguésia 66(4):1085-1113.

Brasil. Instituto Brasileiro de Geografia e Estatística - IBGE. 2004. Mapa de Biomas do Brasil: Primeira Aproximação. Rio de Janeiro, IBGE, 1 mapa, color., Escala 1:5.000.000.

Brasil. Serviço Público Federal - SPF. 2010. Plano de Ação para Prevenção e Controle do Desmatamento e das Queimadas no Cerrado. Serviço Público Federal, Brasília. 159 p.

Bueno, M. L., Neves, D. R. M., Souza, A. F., Oliveira Junior, E., Damasceno Junior, G. A., Pontara, V., Laura, V. A. \& Ratter, J. A. 2013. Influence of edaphic factors on the floristic composition of an area of cerradão in the Brazilian central-west. Acta Botanica Brasilica 27(2):445-455.

Bueno, M. L., Dexter, K. G., Pennington, R. T., Pontara, V., Neves, D. M., Ratter, J. A. \& Oliveira-Filho, A. T. 2018. The environmental triangle of the Cerrado Domain: Ecological factors driving shifts in tree species composition between forests and savannas. Journal of Ecology 106(5):2109-2120.

Bustamante, M. M. C., Martinelli, L. A., Silva, D. A., Camargo, P. B., Klink, C. A., Domingues, T. F. \& Santos, R. V. 2004. ${ }^{15}$ N Abundância natural em plantas lenhosas e solos de savanas da região central do Brasil (Cerrado). Ecological Applications 14(4-supl.):200-213.

Bustamante, M. M. C., Nardoto, G. B., Pinto, A. S., Resende, J. C. F., Takahashi, F. S. C. \& Vieira, L. C. G. 2012. Potential impacts of climate change on biogeochemical functioning of Cerrado ecosystems. Brazilian Journal of Biology 72(3-supl.):655-671.

Cabacinha, C. D. \& Fontes, M. A. L. 2014. Caracterização florística e estrutural de fragmentos de Mata de Galeria da Bacia do Alto Araguaia. Ciência Florestal 24(2):379-390.

Camilotti, D. C., Pagotto, T. C. S. \& Araujo, A. C. 2011. Análise da vegetação arbórea de um remanescente de Cerradão em Bandeirantes, Mato Grosso do Sul, Brasil. Iheringia. Série Botânica 66(1):31-46. 
Campos Filho, E. M. \& Sartorelli, P. A. R. 2015. Guia de identificação de espécies-chave para a restauração florestal na região de Alto Teles Pires, Mato Grosso. The Nature Conservancy, São Paulo. 248 p.

Cândido, J. B., Lima, D. P., Teixeira, P. R., Camargo, M. O., Ferreira, R. Q. S., Souza, P. B. 2018. Síndromes de Dispersão de Espécies Arbustivo-Arbóreas de uma área de Cerrado Sensu Stricto, Gurupi - TO. Global Science Technology 11(1):67-76.

Cândido, J. B., Viana, R. H. O., Morais, I. G., Amorim, M. V. M. \& Souza, P. B. 2019. Chave de identificação dendrológica das espécies mais comuns de uma área de Cerrado sensu stricto, Gurupi, Tocantins. Ciência Florestal 29(1):347-362.

Casella, F. M. \& Silva Júnior, M. C. 2013. Florística, diversidade e estrutura da vegetação arbórea de cerrado sentido restrito e cerradão adjacentes, Parque Ecológico dos Pequizeiros, Distrito Federal. Heringeriana 7(2):127-124.

Castrillon, J. K. I. Silva, C. J., Fernandez, J. R. C. \& Ikeda, A. K. 2011. Avaliação da diversidade arbórea das ilhas do rio Paraguai na região de Cáceres, Pantanal Matogrossense, Brasil. Acta Botanica Brasilica 25(3):672-684.

Cavalcanti, R. B., Cardinot, G.; Ceotto, P. \& Pinheiro, R. S. 2012. Cerrado. In Biomas brasileiros: retratos de um país plural (Scarano, F. R., Santos, I., Martins, A. C. I., Silva, J. M. C., Guimarães, A. \& Mittermeier, R., org.). Casa da Palavra, Rio de Janeiro, p. 57-91.

Costa, C. P., Cunha, C. N. \& Costa, S. C. 2010. Caracterização da flora e estrutura do estrato arbustivo-arbóreo de um cerrado no Pantanal de Poconé, MT. Biota Neotropica 10(3):61-73.

CRIA - Centro de Referência e Informação Ambiental. 2020. Specieslink. Disponível em: http://splink.cria.org.br/. Acessado em 10.07.2020.

Deus, F. F. \& Oliveira, P. E. 2016. Changes in floristic composition and pollination systems in a "Cerrado" community after 20 years of fire suppression. Brazilian Journal of Botany 39(4):1051-1063.

Felfili, J. M. Roitman, I., Medeiros, M. M., \& Sanchez, M. 2011. Procedimentos e Métodos de Amostragem da Vegetação. In Fitossociologia no Brasil: Métodos e estudos de caso (Felfili, J. M. Eisenlohr, P. V., Melo, M. M. R. F., Andrade, L. A., \& Meira Neto, J. A. A., eds.). Universidade Federal de Viçosa, Viçosa, p. 86-121.

Flora do Brasil 2020 em construção. Jardim Botânico do Rio de Janeiro. 2020. Disponível em: http://floradobrasil.jbrj.gov.br/. Acessado em 10.07.2020.

Gama, R. C., Santana, T. T. C., Silva, T. G. N., Andrade, V. C. L. \& Souza, P. B. 2018. Fitossociologia e estrutura diamétrica de um fragmento de Cerrado sensu stricto, Formoso do Araguaia, Tocantins. Revista Verde 13(4):501-506.

Giácomo, R. G., Carvalho, D. C., Pereira, M. G., Souza, A. B. \& Gaui, T. D. 2013. Florística e fitossociologia em áreas de Campo Sujo e Cerrado Sensu Stricto na Estação Ecológica de Pirapitinga - MG. Ciência Florestal 23(1):29-43.

Giácomo, R. G., Pereira, M. G., Carvalho, D. C., Medeiros, V. S., \& Gaui, T. D. 2015. Florística e fitossociologia em áreas de Cerradão e Mata Mesofítica na estação ecológica de Pirapitinga, MG. Floresta e Ambiente 22(3):287-298.

Gomes, B. Z., Martins, F. R. \& Tamashiro, J. Y. 2004. Estrutura do Cerradão e da transição entre Cerradão e floresta Paludícola num fragmento da International Paper do Brasil Ltda., em Brotas, SP. Revista Brasileira de Botânica 27(2):249-262.

Gomes, L. Lenza, E., Maracahipes, L., Marimon, B. S. \& Oliveira, E. A. 2011. Comparações florísticas e estruturais entre duas comunidades lenhosas de cerrado típico e cerrado rupestre, Mato Grosso, Brasil. Acta Botanica Brasilica 25(4):865-875.

International Union for Conservation of Nature - IUCN. 2018. Red List of Threatened Species. Version 2018.2. Disponível em: https://www. iucnredlist.org/. Acessado em 23.12.2019.

JBRJ - Instituto de Pesquisas Jardim Botânico do Rio de Janeiro. Jabot - Banco de Dados da Flora Brasileira. Disponível em: http://jabot. jbrj.gov.br/. Acessado em 10.07.2020.

Köppen, W., Geiger, R. 1928. Klimate der Erde. Gotha: Verlag Justus Perthes. Wall-map $150 \mathrm{~cm} \times 200 \mathrm{~cm}$.

Lewis, G., Schrire, B., Mackinder, B. \& Lock, M. (eds.). 2005. Legumes of the world. Royal Botanic Gardens, Kew. 577 p.

Loschi, R. A., Pereira, J. A. A., Machado, E. L. M., Carlos, L., Gonzaga, A. P. D., Carmo, I. P. \& Gomes, D. J. S. 2013. Variações estruturais e ambientais em um contínuo de Mata de Galeria/Cerrado stricto sensu em Itumirim, MG. Cerne 19(2):213-227.

LPWG (The Legume Phylogeny Working Group). 2013. Legume phylogeny and classification in the 21 st century: Progress, prospects and lessons for other species-rich clades. Taxon 62(2):217-248.

Maria, V. R. B., Maria, F. S. \& Silva, S. M. 2018. Flora arbustivo-arbórea dos planaltos da Bodoquena e de Maracaju, na porção centro-sudoeste do estado do Mato Grosso do Sul, Brasil. Iheringia. Série Botânica 73(supl.):34-52.

Marimon, B.S., Marimon-Junior, B.H., Feldpausch, T.R., Oliveira-Santos, C., Mews, H.A., Lopez-Gonzalez, G., Lloyd, J., Franczak, D.D., Oliveira E.A., Maracahipes, L., Miguel, A., Lenza, E. \& Phillips, O.L. 2014. Disequilibrium and hyperdynamic tree turnover at the forest-cerrado transition zone in southern Amazonia. Plant Ecology \& Diversity 7(1-2):281-292.

Marimon-Junior, B. H., \& Haridasan, M. 2005. Comparação da vegetação arbórea e características edáficas de um cerradão e um cerrado sensu stricto em áreas adjacentes sobre solo distrófico no leste de Mato Grosso, Brasil. Acta Botanica Brasilica 19(4):913-926.

Marques, E. Q., Marimon-Junior, B. H., Marimon, B. S., Matricardi, E. A., Mews, H. A., \& Colli, G. R. 2020. Redefining the CerradoAmazonia transition: implications for conservation. Biodiversity and Conservation 29:1501-1517.

Martins, B. A. A., Ikeda-Castrillon, S. K., Sander, N. L, Olivo-Neto, A. M., Lázaro, W. L. \& Silva, J. C. 2020. Efeito da inundação sobre comunidades arbóreas em floresta poliespecífica na Estação Ecológica de Taiamã (Sítio Ramsar), Pantanal Matogrossense. Research, Society and Development 9(8):e385985808.

Martins-da-Silva, R. C. V., Silva, A. S. L., Fernandes, M. M. \& Margalho, L. F. 2014. Noções Morfológicas e Taxonômicas para Identificação Botânica. Embrapa, Brasília. 111 p.

Matos, M. Q. \& Felfili, J. M. 2010. Florística, fitossociologia e diversidade da vegetação arbórea nas matas de galeria do Parque Nacional de Sete Cidades (PNSC), Piauí, Brasil. Acta Botanica Brasilica 24(2):483-496.

Milan, E., \& Moro, R. S. 2016. O conceito biogeográfico de ecótono. Terr@ Plural, 10(1):75-88

Miller, D. Z. \& Blum, C. T. 2018. Chave dendrológi.ca e caracterização da morfologia vegetativa de espécies lenhosas de Fabaceae em um fragmento de Floresta Ombrófila Mista de Curitiba, PR. Rodriguésia 69(2):787-804.

Morais, R.F., Silva, E.C.S., Metelo, M.R.L. \& Morais, F.F. 2013. Composição florística e estrutura da comunidade vegetal em diferentes fitofisionomias do Pantanal de Poconé, Mato Grosso. Rodriguésia 64(4):775-790.

Neves, S. M. A. S., Nunes, M. C. E. \& Neves, R. J. 2011. Caracterização das condições climáticas de Cáceres/MT Brasil, no período de 1971 a 2009: subsídio às atividades agropecuárias e turísticas municipais. Boletim Goiano de Geografia 31(2):55-68

Oliveira, L. M., Sousa, R. M., Correa, N. E. R.; Santos, A. F. \& Giongo, M. 2018. Florística e síndromes de dispersão de um fragmento de Cerrado ao Sul do estado do Tocantins. Scientia Agraria Paranaensis 17(1):104-111.

Otoni, T. J. O., Pereira, I. M., Oliveira, M. L. R., Machado, E. L. M., Farnezi, M. M. \& Mota, S. L. L. 2013. Componente arbóreo, estrutura fitossociológica e relações ambientais em um remanescente de Cerradão, em Curvelo - MG. Cerne 19(2):201-211.

Pinheiro, E. D. S., \& Durigan, G. 2012. Diferenças florísticas e estruturais entre fitofisionomias do Cerrado em Assis, SP, Brasil. Árvore 36(1):181-193.

Plazas, I. V. C. \& Paula, A. 2016. Chave Dendrológica das Principais Famílias de Importância Florestal. In Anais da IV Semana de Engenharia Florestal da Bahia. Universidade Estadual da Bahia, Vitória da Conquista, p. 1-6.

Pott, A., Oliveira, A. K. M., Damasceno Junior, G. A. \& Silva, J. S. V. 2011. Plant diversity of the Pantanal wetland. Brazilian Journal of Biology 71(1-supl.):265-273.

Procópio, L.C. \& Secco, R.S. 2008. A importância da identificação botânica nos inventários florestais: o exemplo do "tauari" (Couratari spp. e Cariniana spp. - Lecythidaceae) em duas áreas manejadas no estado do Pará. Acta Amazonica 38(1):31-44. 
R Core Team. 2017. R: A language and environment for statistical computing. R Foundation for Statistical Computing, Vienna, Austria.

Ribeiro, J. F. \& Walter, B. M. T. 2008. As principais fitofisionomias do Bioma Cerrado. In Cerrado: ecologia e flora (Sano, S.M., Almeida, S.P. \& Ribeiro, J.F., eds.). Embrapa Cerrados/Embrapa Informação Tecnológica, Planaltina, 151-212 p.

Rodrigues, R. F., \& Araújo, G. M. (2013). Estrutura da vegetação e características edáficas de um cerradão em solo distrófico e em solo mesotrófico no Triângulo Mineiro. Bioscience Journal 29(6):20132029.

Sabino, F. G. S., Cunha, M. C. L. \& Santana, G. M. 2016. Estrutura da vegetação em dois fragmentos de Caatinga antropizada na Paraíba. Floresta e Ambiente 23(4):487-497.

Saueressig, D. 2017. Manual de dendrologia. Editora Plantas do Brasil, Irati. $150 \mathrm{p}$.

Saueressig, D., Saueressig, A. \& Inoue, M. T. 2009. SIDOL - Sistema de Identificação Dendrológica On-line. Ambiência 5(1):117-133.

Saueressig, D. 2018. Manual de dendrologia: o estudo das árvores. Editora Plantas do Brasil, Irati. 304 p.

Silva, R. A., Paixão, E. C., Cunha, C. N. \& Finger, Z. 2016. Fitossociologia da comunidade arbórea de Cerrado Sensu Stricto do Parque Nacional da Chapada dos Guimarães. Nativa 4(2):82-86.
Siqueira, A.S., Araújo, G.M. \& Schiavini, I. 2006. Caracterização florística da Reserva Particular do Patrimônio Natural (RPPN) Fazenda Carneiro, Lagamar, MG, Brasil. Biota Neotropica 6(3):-.

Solórzano, A., Pinto, J. R. R., Felfili, J. M. \& Hay, J. D. V. 2012. Perfil florístico e estrutural do componente lenhoso em seis áreas de cerradão ao longo do bioma Cerrado. Acta Botanica Brasilica 26(2):328-341.

Souza, P. B., Saporetti Junior, A. W., Soares, M. P., Viana, R. H. O., Camargos, V. L. \& Meira Neto, J. A. A. 2010. Florística de uma área de cerradão na floresta nacional de Paraopeba - Minas Gerais. Cerne 16(1):86-93.

Souza, V. C. \& Lorenzi, H. 2012. Botânica Sistemática: guia ilustrado para identificação das famílias de Fanerógamas nativas e exóticas no Brasil, baseado em APG III. Instituto Plantarum, Nova Odessa. 768 p.

Souza, V. C., Flores, T. B., Colletta, G. D. \& Coelho, R. L. G. 2018. Guia das Plantas do Cerrado. Taxon Brasil, Piracicaba. 583 p.

Stefanello, D., Fernandes-Bulhão, C. \& Martins, S. V. 2009. Síndromes de Dispersão de Sementes em Três Trechos de Vegetação Ciliar (Nascente, Meio e Foz) ao longo do Rio Pindaíba, MT. Árvore 33(6):1051-1061.

Tropicos. Saint Louis: Missouri Botanical Garden. 2019. Disponível em: http://www.tropicos.org/. Acessado em 15.12.2019.

Zanatta, M.R.V., Kuhlmann, M., Cota, M. R.C., Santos, A.B.P., \& Proença, C.E.B. 2015. Chave interativa ilustrada para famílias de angiospermas do bioma Cerrado. Heringeriana 9(2):91-112. 